\title{
A Case Report Of Suppurative Pericarditis
}

\author{
By Henry A Mayala, Levi Kwarisiima, Robert Mvungi, Peter Kisenge, \\ Nyangasa, Nyawawa, Kawajika
}

\begin{abstract}
Background: Suppurative pericarditis is defined as an infection of the pericardial space that produces pus that is found on gross examination of the pericardial sac or on tissue microscopy. Bacterial infections of the pericardium are relatively uncommon but are much more likely to produce purulent effusions and to proceed to cardiac tamponade and pericardial constriction ${ }^{1}$.
\end{abstract}

Case presentation: In this case report a 57 year old lady with confirmed diagnosis SLE on treatment (prednisolone and methotrexate) was admitted at cardiovascular medicine department of Muhimbili national hospital(MNH) with the chief complains of cough, chest pain and difficulty in breathing, with associated history of weight loss. Clinical and imaging findings are suggestive of suppurative pericarditis and it was confirmed and managed by surgical pericardial drainage of $700 \mathrm{mls}$ of pus.

Conclusion: purulent/suppurative pericarditis should be suspected in ill patients with thoracic infections, patients will often be febrile and tachycardic without a doubt optimal care of these complicated infections requires a multidisciplinary approach ${ }^{2}$.

Keywords: purulent, suppurative pericarditis, bacteria, CT-scan, pericardial drainage

\section{INTRODUCTION}

Purulent pericarditis is defined as an infection of the pericardial space that produces pus that is found on gross examination of the pericardial sac or on tissue microscopy.Bacterial infections of the pericardium are relatively uncommon but are much more likely to produce purulent effusions and to proceed to cardiac tamponade and pericardial constriction1. Purulent pericarditis occurs almost exclusively as a secondary infection in patients with AIDS, and those undergoing hemodialysis, thoracic surgery and chemotherapy.Numerous microbes are capable of causing pericardial infection; viral agents are common identified cause of pericarditis. Viral and idiopathic pericarditis accounts for $90 \%$ of cases of acute pericarditis. Coxsackie virus B, adenovirus, Echoviridae and Retroviridae are usually responsible. Bacterial pericarditis is not typically a primary infection but is almost exclussively a complication from an underlying infection. In pre-antibiotic era, patients most frequently developed bacterial pericarditis due to pneumonia with empyema and most common organism was streptoccocus pneumoniae. In the antibiotic era, the most common causative organism was staphylococcus aureus. Recent studies have noted a trend towards involvement of more diverse microbes and anaerobes have been reported as a common cause of pericardial infections. Fungal and tuberculosis also cause purulent pericarditis. The recognized risk factors for purulent pericarditis include: advanced age, diabetes mellitus, untreated infection(pneumonia), extensive burns, and immunosuppressed state. ${ }^{1}$

Case Presentation: We present a case report of a 57 years old female with confirmed diagnosis SLE on treatment (prednisolone and methotrexate) admitted at Cardiovascular department of Muhimbili National Hospital with the chief complains of cough, chest pain, and difficulty in breathing, with the positive history of loss of weight. she is not diabetic nor hypertensive. She has been on treatment of SLE (Prednisolone and Methotrexate) for 4 years

on admission the following investigation were done: chest $\mathrm{x}$-ray, ECG, ,Echo, chest CT-scan, renal function, liver function and lipid profile, sputum for AFB,FBP 


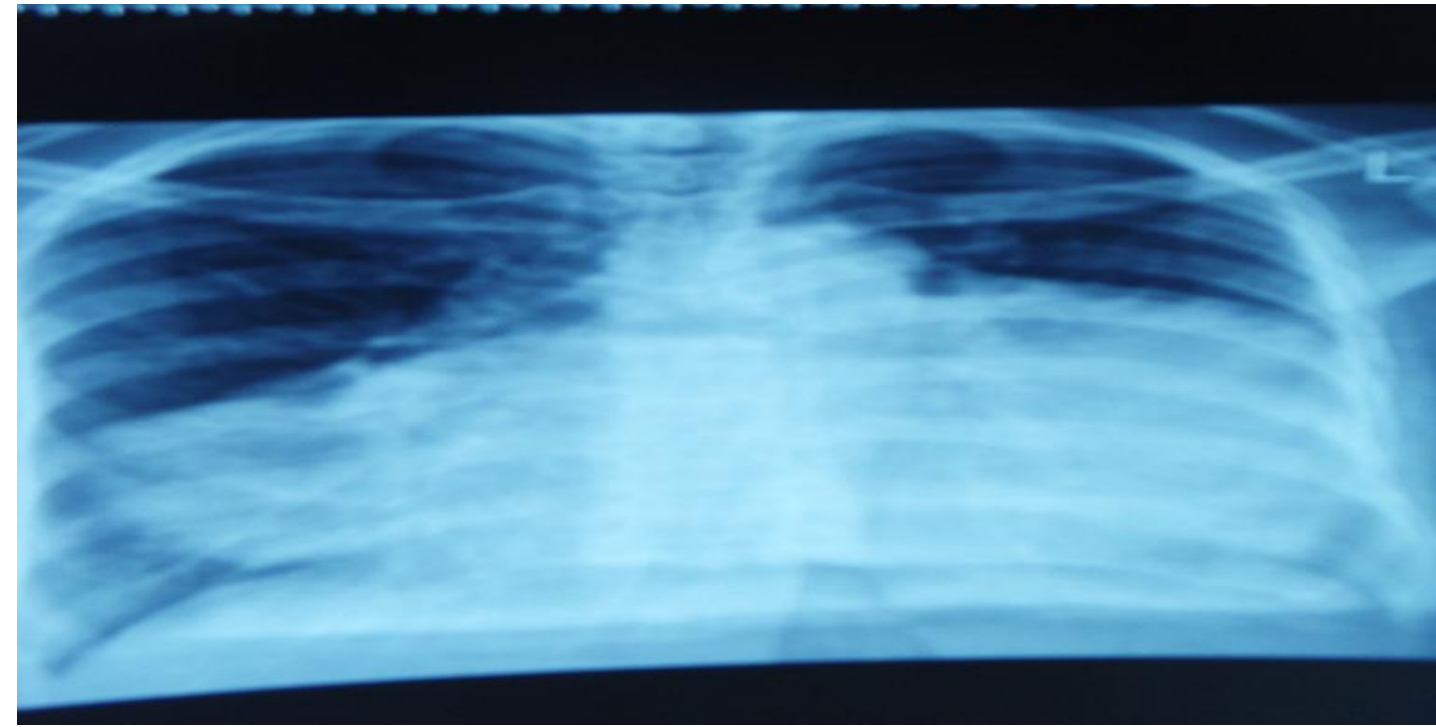

FIGURE 1: A CHEST X-RAY - showing abnormal cardiac silhouette-flask shaped appearance

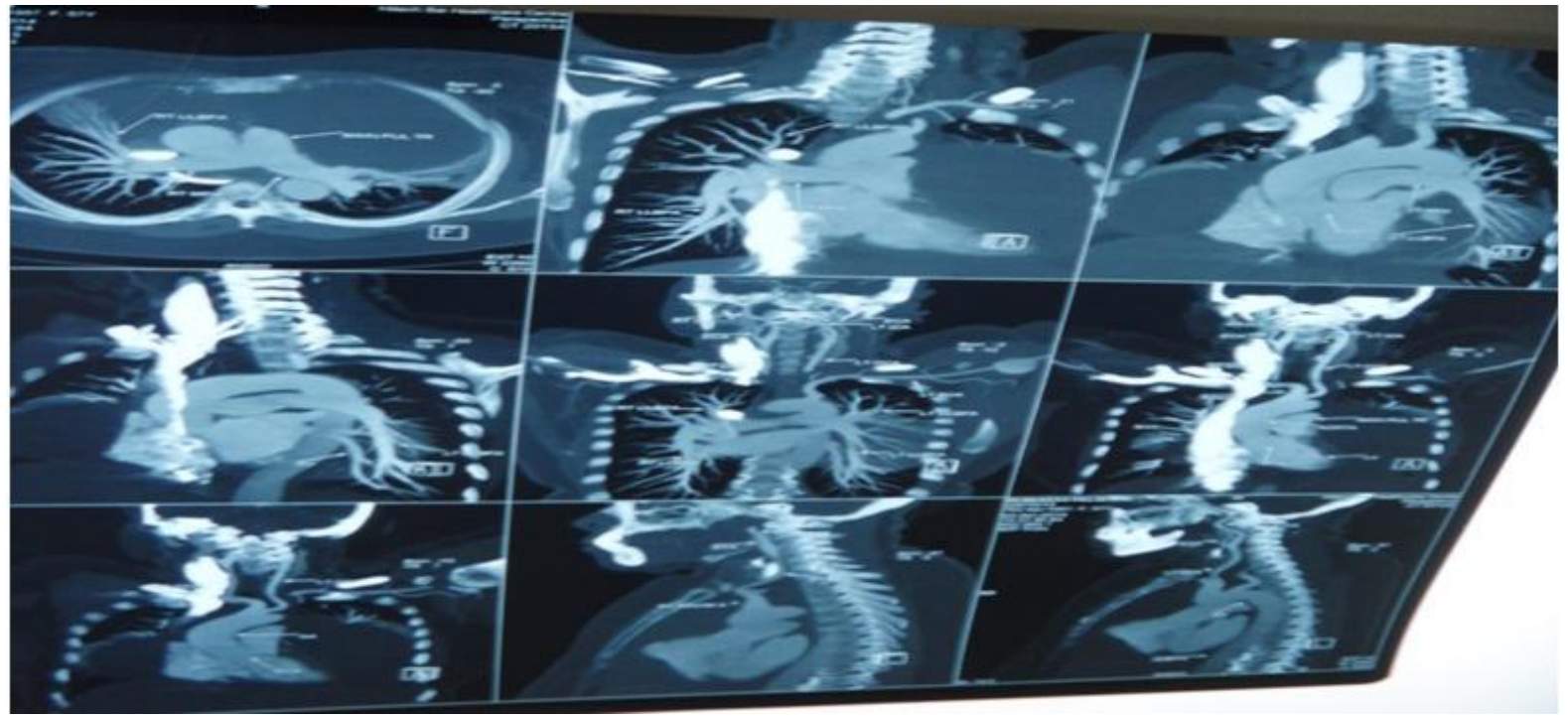

FIGURE 2: CHEST CT-ANGIOGRAPHY- showing pericardial effusion

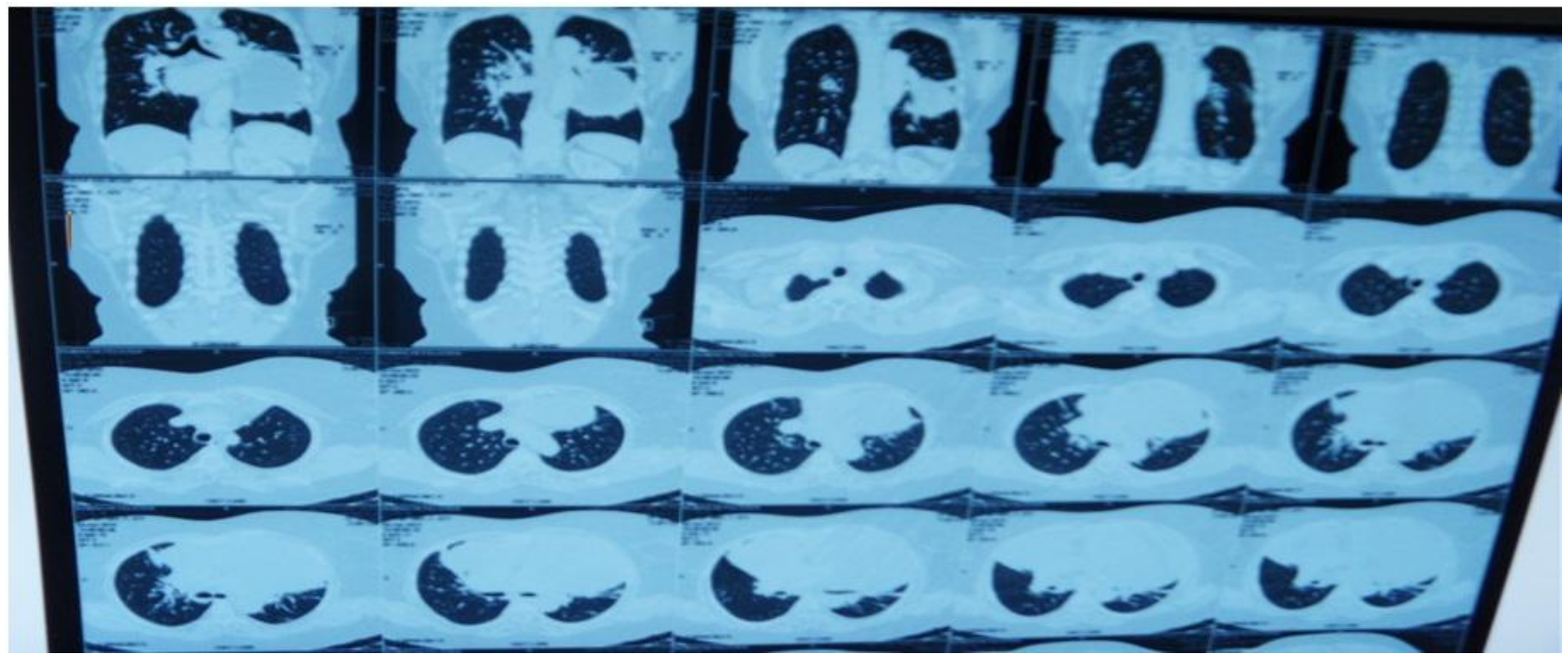



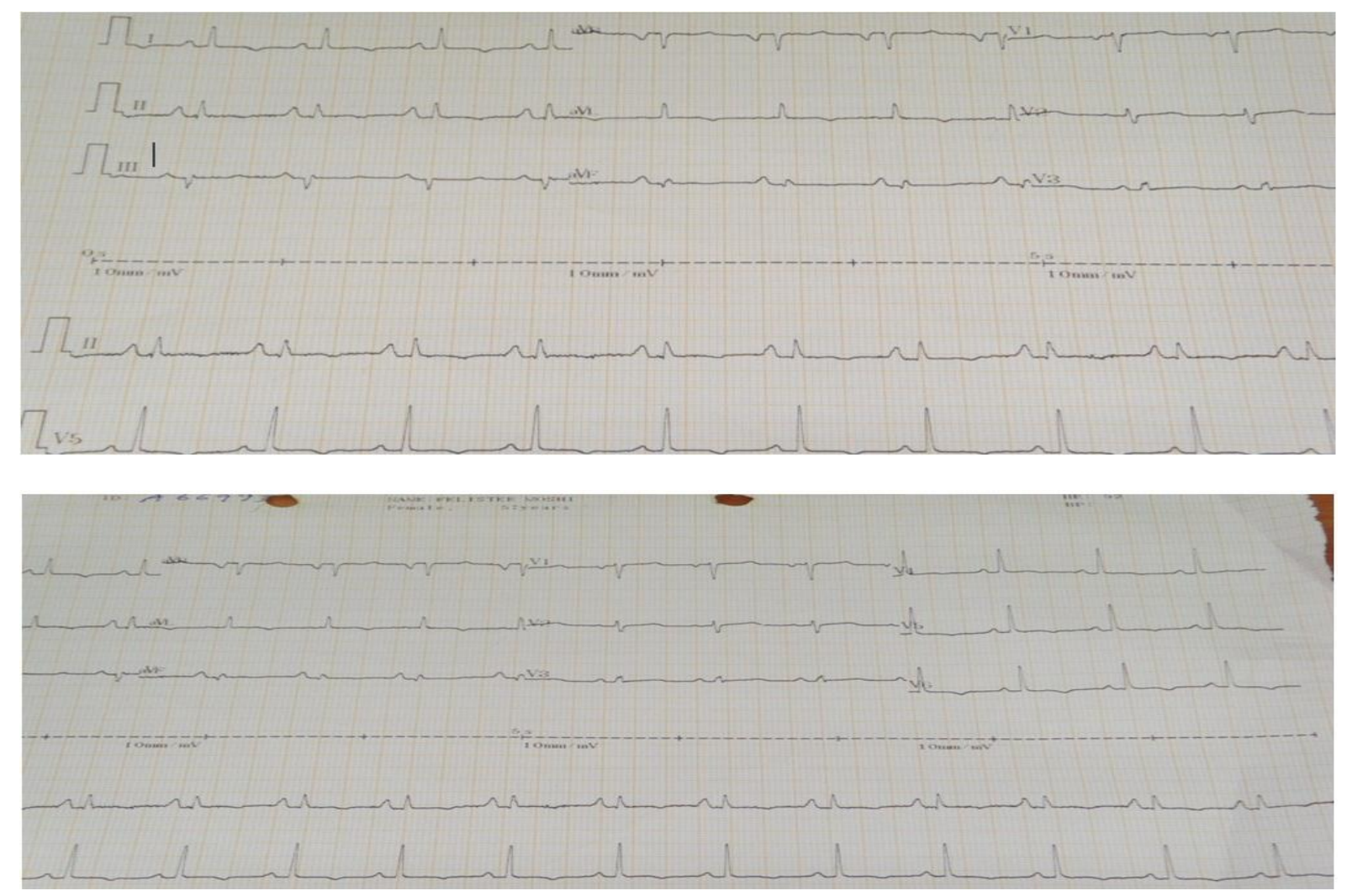

FIGURE 4: ECG revealed non specific ST-changes and right atrial enlargement

Echo revealed a swinging heart with a large pericardial effusion

Renal function test, liver function test and lipid profile were normal.

Pus culture - there was no growth

Sputum for AFB- was negative

FBP-showed leucocytopenia and anemia

\section{DISCUSSION:}

Purulent pericarditis is an infrequent, but important complication of infective illness, which if diagnosed early has a good prognosis. The incidence of the condition is probably increasing particularly in the immune-compromised group of patients. Classical symptoms and signs are often absent and a high index of awareness is required to diagnose the condition ${ }^{7}$. Our patient presented with some symptoms cough, chest pain and difficulty in breathing but we had to have a high index of suspicion to diagnose it with atypical ECG presentation.

Thus this case report is so significant because of how it presented and managed. Our patient underwent a surgical pericardial drainage of $700 \mathrm{mls}$ of pus, which was successful. Currently our patient is discharged, with vital signs stable, on the following medication: Tazact and metronidazole and breathing exercise (respiratory physiotherapy).

\section{REFERENCES:}

[1] www.turner-white.com (hospital physician)- Jan 2008, shiber pp.9-17,45

[2] Lange RA, hillis L.D. clinical practice. acute pericarditis in N Engl J Med 2005; 352:1163

[3] Goodman L.J. purulent pericarditis. Curr Treat options cardiovasc Med 2000; 2:343-50

[4] Spodick DH. The pericardium: a comprehensive text book. Newyork: M.bekker, 1997

[5] www.emedicine.medscape.com

[6] www.ncbi.nlm.nih.gov

[7] Annals of surgery (Ann surg. sep 1936; 104(3): 365-372 by James Harry Heyl

[8] Textbook of Mayo clinic Internnal medicine

[9] Rubin RH, moelling RC, Jr.Clinical microbiological and therapeutic aspects of purulent pericarditis. AM J med. 1975 Jul:59(1):68-78. (Pubmed) 